\title{
Sieve of Prime Numbers Using Algorithms
}

\section{Stelian Liviu B*}

Independent Researcher, Israel

\begin{abstract}
This study suggests grouping of numbers that do not divide the number 3 and/or 5 in eight columns. Allocation results obtained from multiplication of numbers is based on column belonging to him. If in the Sieve of Eratosthenes the majority of multiplication of prime numbers result in a results devoid of practical benefit (numbers divisible by 2 , 3 and/or 5), in the sieve of prime numbers using algorithms, each multiplication of prime number gives a result in a number not divisible to 2,3 and/or 5 .
\end{abstract}

Keywords: Column; Factor; Position; Sieve; Termination

\section{Introduction}

\section{Sieve of prime numbers using algorithms}

This paper deals with the study of odd numbers that cannot be divided with 3 and/or 5 by grouping them in eight columns, as follows:

The multiplication versions are in number of 36 , their results being allocated according to columns, explained in Table 1.

\section{Position Calculus}

From the result of multiplying two numbers subtract the number assigned at position zero of the column namely one of the numbers $\mathrm{i}(\mathrm{p} 0)$ : 7-11-13-17-19-23-29-31, the result is divided by 30. Integer obtained indicates the position of that number considering its column origin $[1,2]$.

\section{Formulas for determining the position}

Position occupied by the result of the multiplication between

\begin{tabular}{|l|l|l|l|l|l|l|l|l|}
\hline Col.1=Col. & $1 \times 8$ & $2 \times 4$ & $3 \times 5$ & & & $6 \times 7$ & & \\
\hline Col.2=Col. & $1 \times 6$ & $2 \times 8$ & $3 \times 4$ & & $5 \times 7$ & & & \\
\hline Col.3=Col. & $1 \times 5$ & $2 \times 6$ & $3 \times 8$ & $4 \times 7$ & & & & \\
\hline Col.4=Col. & $1 \times 2$ & & $3 \times 7$ & $4 \times 8$ & $5 \times 6$ & & & \\
\hline Col.5=Col. & $1 \times 1$ & $2 \times 7$ & $3 \times 3$ & $4 \times 4$ & $5 \times 8$ & $6 \times 6$ & & \\
\hline Col.6=Col. & $1 \times 7$ & $2 \times 3$ & & $4 \times 5$ & & $6 \times 8$ & & \\
\hline Col.7=Col. & $1 \times 4$ & $2 \times 5$ & $3 \times 6$ & & & & $7 \times 8$ & \\
\hline Col. $8=$ Col. & $1 \times 3$ & $2 \times 2$ & & $4 \times 6$ & $5 \times 5$ & & $7 \times 7$ & $8 \times 8$ \\
\hline
\end{tabular}

Table 1: Multiplication versions are in number of 36 .

\begin{tabular}{|c|c|c|c|c|c|c|c|c|}
\hline Position & $\mathbf{1}$ & $\mathbf{2}$ & $\mathbf{3}$ & $\mathbf{4}$ & $\mathbf{5}$ & $\mathbf{6}$ & $\mathbf{7}$ & $\mathbf{8}$ \\
\hline $\mathbf{0}$ & 7 & 11 & 13 & 17 & 19 & 23 & 29 & 31 \\
\hline $\mathbf{1}$ & 37 & 41 & 43 & 47 & 49 & 53 & 59 & 61 \\
\hline $\mathbf{2}$ & 67 & 71 & 73 & 77 & 79 & 83 & 89 & 91 \\
\hline $\mathbf{3}$ & 97 & 101 & 103 & 107 & 109 & 113 & 119 & 121 \\
\hline
\end{tabular}

Table 2: Odd numbers that cannot be divided with 3 and/or 5

\begin{tabular}{|c|c|c|c|c|c|c|c|c|}
\hline $7+31$ & $5+23$ & $4+19$ & $2+11$ & $1+7$ & $6+29$ & $3+17$ & $2+13$ & $+37 n$ \\
\hline $6+17$ & $11+31$ & $8+23$ & $2+7$ & $10+29$ & $4+13$ & $6+19$ & $3+11$ & $+41 n$ \\
\hline $8+19$ & $7+17$ & $13+31$ & $12+29$ & $5+13$ & $4+11$ & $9+23$ & $2+7$ & $+43 n$ \\
\hline $6+11$ & $7+13$ & $16+29$ & $17+31$ & $9+17$ & $10+19$ & $3+7$ & $12+23$ & $+47 n$ \\
\hline $\mathbf{8 + 1 3}$ & $\mathbf{1 8 + 2 9}$ & $\mathbf{4 + 7}$ & $\mathbf{1 4 + 2 3}$ & $\mathbf{1 9 + 3 1}$ & $\mathbf{1 0 + 1 7}$ & $\mathbf{6 + 1 1}$ & $\mathbf{1 1 + 1 9}$ & $+\mathbf{4 9 n}$ \\
\hline $22+29$ & $5+7$ & $8+11$ & $14+19$ & $17+23$ & $23+31$ & $9+13$ & $12+17$ & $+53 n$ \\
\hline $22+23$ & $18+19$ & $16+17$ & $12+13$ & $10+11$ & $6+7$ & $29+31$ & $27+29$ & $+59 n$ \\
\hline $7+7$ & $11+11$ & $13+13$ & $17+17$ & $19+19$ & $23+23$ & $29+29$ & $31+31$ & $+61 n$ \\
\hline
\end{tabular}

Table 3: Position occupied $\mathrm{p} 1$ as a result of multiplication of numbers $\mathrm{i}$.

\begin{tabular}{|c|c|c|c|c|c|c|c|c|}
\hline $7+31 \times 2$ & $5+23 \times 2$ & $4+19 \times 2$ & $2+11 \times 2$ & $1+7 \times 2$ & $6+29 \times 2$ & $3+17 \times 2$ & $2+13 \times 2$ & $+67 n$ \\
\hline $6+17 \times 2$ & $11+31 \times 2$ & $8+23 \times 2$ & $2+7 \times 2$ & $10+29 \times 2$ & $4+13 \times 2$ & $6+19 \times 2$ & $3+11 \times 2$ & $+71 n$ \\
\hline $8+19 \times 2$ & $7+17 \times 2$ & $13+31 \times 2$ & $12+29 \times 2$ & $5+13 \times 2$ & $4+11 \times 2$ & $9+23 \times 2$ & $2+7 \times 2$ & $+73 n$ \\
\hline $\mathbf{6 + 1 1 \times 2}$ & $\mathbf{7 + 1 3 \times 2}$ & $\mathbf{1 6 + 2 9 \times 2}$ & $\mathbf{1 7 + 3 1 \times 2}$ & $\mathbf{9 + 1 7 \times 2}$ & $\mathbf{1 0 + 1 9 \times 2}$ & $\mathbf{3 + 7 \times 2}$ & $\mathbf{1 2 + 2 3 \times 2}$ & $+\mathbf{7 7 n}$ \\
\hline $8+13 \times 2$ & $18+29 \times 2$ & $4+7 \times 2$ & $14+23 \times 2$ & $19+31 \times 2$ & $10+17 \times 2$ & $6+11 \times 2$ & $11+19 \times 2$ & $+79 n$ \\
\hline $22+29 \times 2$ & $5+7 \times 2$ & $8+11 \times 2$ & $14+19 \times 2$ & $17+23 \times 2$ & $23+31 \times 2$ & $9+13 \times 2$ & $12+17 \times 2$ & $+83 n$ \\
\hline $22+23 \times 2$ & $18+19 \times 2$ & $16+17 \times 2$ & $12+13 \times 2$ & $10+11 \times 2$ & $6+7 \times 2$ & $29+31 \times 2$ & $27+29 \times 2$ & $+89 n$ \\
\hline $\mathbf{7 + 7 \times 2}$ & $\mathbf{1 1 + 1 1 \times 2}$ & $\mathbf{1 3 + 1 3 \times 2}$ & $\mathbf{1 7 + 1 7 \times 2}$ & $\mathbf{1 9 + 1 9 \times 2}$ & $\mathbf{2 3 + 2 3 \times 2}$ & $\mathbf{2 9 + 2 9 \times 2}$ & $\mathbf{3 1 + 3 1 \times 2}$ & $+\mathbf{9 1 n}$ \\
\hline
\end{tabular}

Table 4: Positions of $\mathrm{p} 1$ are used to calculate $\mathrm{p} 2, \mathrm{p} 3, \mathrm{p} 4$.

\begin{tabular}{|c|c|c|c|c|c|c|c|c|}
\hline $\mathbf{1}$ & $\mathbf{2}$ & $\mathbf{3}$ & $\mathbf{4}$ & $\mathbf{5}$ & $\mathbf{6}$ & $\mathbf{7}$ & $\mathbf{8}$ & $\mathbf{9}$ \\
\hline 7 & 5 & 4 & 2 & 1 & 6 & 3 & 2 & $+7 \mathrm{n}$ \\
\hline 6 & 11 & 8 & 2 & 10 & 4 & 6 & 3 & $+11 \mathrm{n}$ \\
\hline 8 & 7 & 13 & 12 & 5 & 4 & 9 & 2 & $+13 \mathrm{n}$ \\
\hline 6 & 7 & 16 & 17 & 9 & 10 & 3 & 12 & $+17 \mathrm{n}$ \\
\hline 8 & 18 & 4 & 14 & 19 & 10 & 6 & 11 & $+19 \mathrm{n}$ \\
\hline 22 & 5 & 8 & 14 & 17 & 23 & 9 & 12 & $+23 \mathrm{n}$ \\
\hline 22 & 18 & 16 & 12 & 10 & 6 & 29 & 27 & $+29 \mathrm{n}$ \\
\hline 7 & 11 & 13 & 17 & 19 & 23 & 29 & 31 & $+31 \mathrm{n}$ \\
\hline
\end{tabular}

Table 5: Position occupied $p 0$ as a result of multiplication of numbers $i(p 0)$ and all the numbers.

numbers $i(p 0), i(p 1), i(p 2), \ldots, i(p n)$, with all the numbers in Table 2. Position occupied $\mathrm{p} 1$ as a result of multiplication of numbers $\mathrm{i}(\mathrm{p} 1)$ and all the numbers in Table 3;

Positions of $\mathrm{p} 1$ are used to calculate $\mathrm{p} 2, \mathrm{p} 3, \mathrm{p} 4, \ldots . ., \mathrm{pn}$ multiplying $\mathrm{i}(\mathrm{p} 0)$, positions occupied $\mathrm{p} 2$ as a result of multiplication of numbers $\mathrm{i}(\mathrm{p} 2)$ and all the numbers in Table 4;

\section{Calculation algorithm}

- Fill in Table 1 with all the numbers to be tested if they are prime number;

- Write all numbers under test, in order of their increasing in column 9,as shown in Table 5;

- Fill p0 formulas in Table 5;

*Corresponding author: Stelian Liviu B, Independent researcher, Israel, Tel: 9720545723072; E-mail: stelibarar@yahoo.com

Received February 29, 2016; Accepted April 29, 2016; Published May 05, 2016

Citation: Stelian Liviu B (2016) Sieve of Prime Numbers Using Algorithms. J Phys Math 7: 170. doi:10.4172/2090-0902.1000170

Copyright: $\odot 2016$ Stelian Liviu B. This is an open-access article distributed under the terms of the Creative Commons Attribution License, which permits unrestricted use, distribution, and reproduction in any medium, provided the original author and source are credited. 
- Mark all numbers divisible in Table 1 by the formulas of $\mathrm{p} 0$;

- Eliminates all the numbers in column 9 Table 2 that were marked in Table 1 according to the formulas of p0;

- Fill formulas of p1 Table 2; number 49 was removed according to Table 1 no longer consider;

- Repeat the operations made in step 4 and 5 according to the formulas $\mathrm{p} 1$;

- Fill formulas of p2 Table 2 and repeat the operations in step 4 and 5. Numbers not eliminated in column 9 Table 2 are prime numbers.

In column 9 we register numbers under test up to $\mathrm{P}(\max )$. Maxim position calculation is the integer number of the maximum number being tested radical divided by 30 [2-4].

Formulas belonging composite numbers are omitted. The algorithm uses formulas primes numbers squared correlating $n=0,1,2,3, \ldots$. With Pn.

Using the tables respecting the above algorithm complexity is much smaller, any multiple of prime number (which represents the number of position) has corresponding number is compound odd number and not divisible by 3 and/or 5 .

Example: Determination of prime numbers up to $\mathrm{N}=1001$.

In parentheses are the numbers corresponding to position past according to column.

Divisibility by 7 :

Col.1: 7+7n=7(217) $-14(427)-21(637)-28(847)$

Col.2: $5+7 \mathrm{n}=5(161)-12(371)-19(581)-26(791)-33(1001)$

Col.3: $4+7 \mathrm{n}=4(133)-11(343)-18(553)-25(763)-32(973)$

Col.4: $2+7 n=2(77)-9(287)-16(497)-23(707)-30(917)$

Col.5: $1+7 \mathrm{n}=1(49)-8(259)-15(469)-22(679)-29(889)$

Col.6: $6+7 n=6(203)-13(413)-20(623)-27(833)$

Col.7=3+7n=3(119) - 10(329) - 17(539) - 24(749) - 31(959)

Col. $8=2+7 n=2(91)-9(301)-16(511)-23(321)-30(931)$

Divisibility by 11 :

Col.1=6+11n=6(187) $-17(517)-28(847)$

Col.2=11+11n=11(341) $-22(671)-33(1001)$

Col.3=8+11n=8(253) $-19(583)-30(913)$

Col.4=2+11n=2(77) $-13(407)-24(737)$

Col.5=10+11n=10(319) $-21(649)-32(979)$

Col.6=4+11n=4(143) $-15(473)-26(803)$

Col. $7=6+11 \mathrm{n}=6(209)-17(539)-28(869)$

Col. $8=3+11 n=3(121)-14(451)-25(781)$

Divisibility by 13 :

Divisibility by 17 :

Col. $1=8+13 n=8(247)-21(637)$

Col. $1=6+17 n=6(187)-23(697)$
Col. $2=7+13 n=7(221)-20(611)-33(1001)$

Col.2 $=7+17 \mathrm{n}=7(221)-24(731)$

Col. $3=13+13 n=13(403)-26(793)$

Col. $3=16+17 \mathrm{n}=16(493)$

Col. $4=12+13 n=12(377)-25(767)$

Col. $4=17+17 \mathrm{n}=17(527)$

Col. $5=5+13 n=5(169)-18(559)-31(949)$

Col. $5=9+17 n=9(289)-26(799)$

Col. $6=4+13 n=4(143)-17(533)-30(923)$

Col.6=10+17n=10(323) $-27(833)$

Col.7=9+13n=9(299) $-22(689)$

Col. $7=3+17 n=3(119)-20(629)$

Col. $8=2+13 n=2(91)-15(481)-28(871)$

Col. $8=12+17 \mathrm{n}=12(391)-29(901)$

Divisibility by 19 :

Divisibility by 23 :

Col.1 $=8+19 \mathrm{n}=8(247)-27(817)$

Col. $1=22+23 n=22(667)$

Col. $2=18+19 n=18(551)$

Col.2 $=5+23 n=5(161)-28(851)$

Col. $3=4+19 n=4(133)-23(703)$

Col. $3=8+23 n=8(253)-31(943)$

Col.4=14+19n=14(437)

Col. $4=14+23 n=14(437)$

Col.5=19+19n=19(589)

Col. $5=17+23 \mathrm{n}=17(529)$

Col. $6=10+19 n=10(323)-29(893)$

Col. $6=23+23 n=23(713)$

Col.7=6+19n=6(209) $-25(779)$

Col. $7=9+23 n=9(299)-32(789)$

Col. $8=11+19 n=11(361)-30(961)$

Col. $8=12+23 n=12(391)$

Divisibility By 29:

Divisibility by 31 :

Col. $1=22+29 n=22(667)$

Col. $1=7+31=7(217)$

Col.2 $=18+29 \mathrm{n}=18(551)$

Col. $2=11+31=11(341)$

Col. $3=16+29 n=16(493)$

Col. $3=13+31 \mathrm{n}=13(403)$

Col. $4=12+29 \mathrm{n}=12(377)$

Col. $4=17+31 \mathrm{n}=17(527)$

Col. $5=10+29 n=10(319)$

Col.5=19=31n=19(589)

Col. $6=6+29 n=6(203)$

Col. $6=23+31 \mathrm{n}=23(713)$

Col. $7=29+29 n=29(899)$

Col. $7=29+31 \mathrm{n}=29(899)$ 
Col. $8=27+29 n=27(841)$

Col. $8=31+31 \mathrm{n}=31(961)$

Numbers not eliminated are prime numbers

\section{Application: The Factorial Multiplying or the Method of Determining if a Number is Prime up to a Given Number}

The method of grouping odd numbers according to Table 1, allows checking whether a number is prime according to the last two or five digits of position the number.

\section{For termination two digits}

The calculation algorithm is:

Step 1: Determine the position number and column it belongs;

Step 2: Last two digits of the calculated number indicates the termination position of tested number;

Step 3: Determine factors for termination and column number tested. I have illustrated the calculation of factors termination 10 , column 1. Once calculated these factors can be used to determine of any prime numbers that belongs to the column 1, termination 10 .

Step 4: It performs testing divisibility of a number with multiples of 3000 plus pairs of numbers factorial group to which it belongs termination corresponding column number tested.

We assign factorial group for multiplying operation positions from 0-99, as in Table 1, numbers between 7-3.001 grouped in columns. The position occupied by the result of the multiplication between any two numbers in the factorial group is a maximum six digit number. The last two digits of the number shows the termination, the rest of maximum four digits is the factor and which the position will be calculated for those termination belonging to specific column $[5,6]$.

I1 and I2 are two numbers higher than the numbers belonging to factorial group.

Position obtained by multiplying the numbers is determined by formula:

$\mathrm{P}=\mathrm{n} 2 \times \mathrm{i} 1(\mathrm{f})+\mathrm{n} 1 \times \mathrm{i} 2+\mathrm{F}$, followed by $\mathrm{T}$

Or, $=\mathrm{n} 1 \times \mathrm{i} 2(\mathrm{f})+\mathrm{n} 2 \times \mathrm{i} 1+\mathrm{F}$, followed by $\mathrm{T}$

Where:

n1, n2: represents multiples of 3000 corresponding of i1(f), respectively i2(f);

i1 (f), i2 (f): represents the corresponding numbers of i1 and i2 in factorial group;

F - Factor

$\mathrm{T}$ - Termination

Be: $32999 \times 32693=1078836307$ 612

$\mathrm{P}=(1078836307-7): 30=35961210$ col. $1 \mathrm{~T}=10 \mathrm{p}($ without $\mathrm{T})=359$

Factor calculation and termination:

$2999 \times 2693=(8076307-7): 30=269210 ; \mathrm{F}=2692 \mathrm{~T}=10$

$\mathrm{P}=10 \times 2999+10 \times 32693+\mathrm{F}$, followed by $\mathrm{T}$
$=10 \times 2693+10 \times 32999+F$, followed by $\mathrm{T}$

We calculate all the factors column 1 , termination 10 . The four types of multiplication corresponding col. 1 between numbers belonging to factor group, generates 400 factors with T.10, as follows:

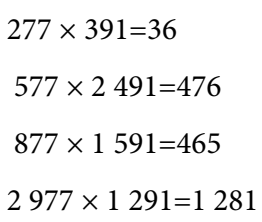

Or,

$11 \times 1937=741 \times 227=371 \times 2117=50$

$311 \times 2837=294341 \times 1127=128371 \times 17=2$

$611 \times 737=150641 \times 2027=433671 \times 917=205$

$2711 \times 1037=9372741 \times 2327=21262771 \times 1217=1124$

$101 \times 1607=54131 \times 1697=74161 \times 2387=128$

$401 \times 2507=335431 \times 2597=374461 \times 287=44$

$701 \times 407=95731 \times 497=121761 \times 1187=3011$

$2801 \times 707=6602831 \times 797=7522861 \times 1487=1418$

$191 \times 677=43221 \times 2567=189251 \times 2057=172$

$491 \times 1577=258521 \times 467=81551 \times 2957=543$

$791 \times 2477=653821 \times 1367=374851 \times 857=243$

$2891 \times 2777=26762921 \times 1667=16232951 \times 1157=1138$

$281 \times 2147=201$

$581 \times 47=9$

$881 \times 947=278$

$2981 \times 1247=1239$

Or,

$19 \times 1753=1149 \times 1843=3079 \times 1333=35$

$319 \times 2653=282349 \times 2743=319379 \times 2233=282$

$619 \times 553=114649 \times 643=139679 \times 133=30$ 


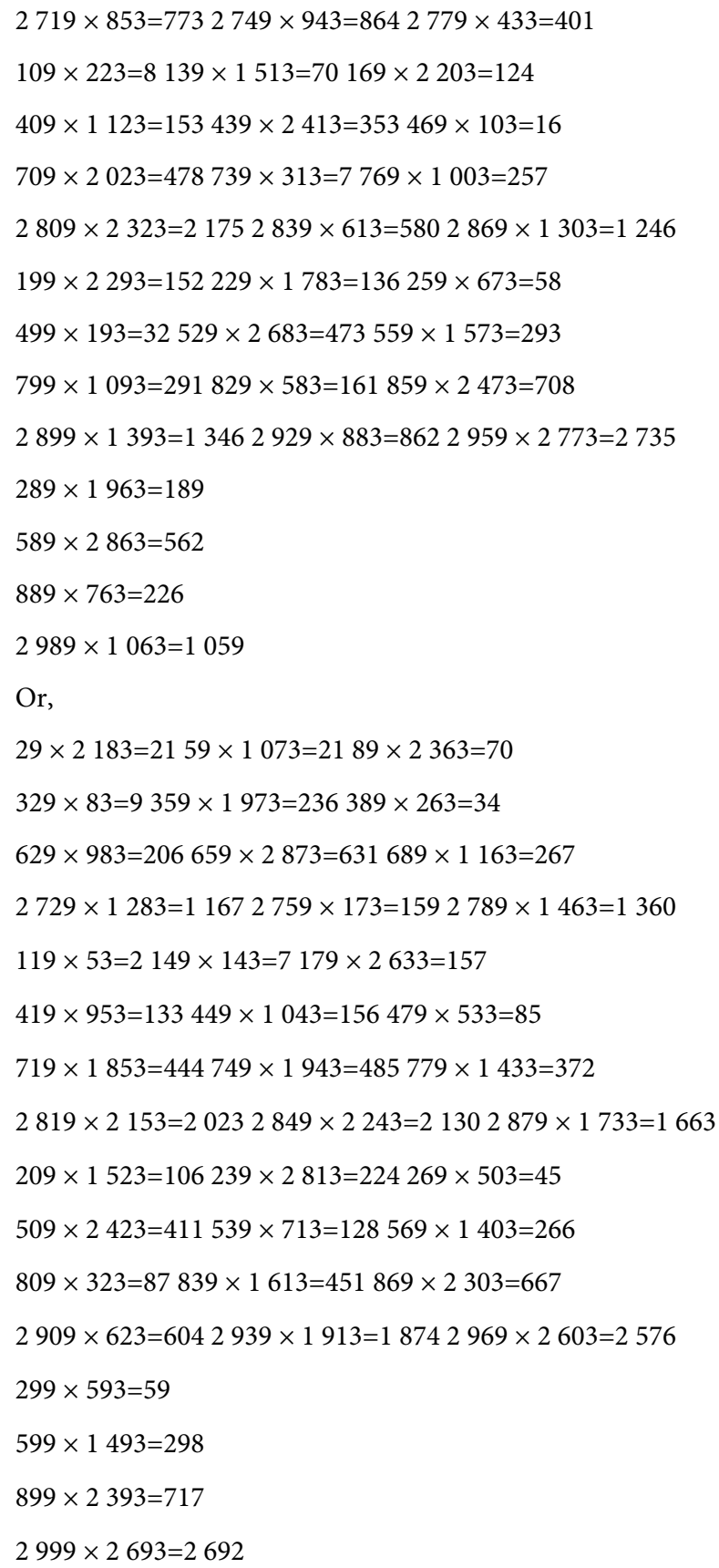

Grouping numbers from left of multiplying operation according to the above model, in this case numbers on the right have a constant growth rate, which allows for relatively simple determination of them. Perform tests to see if number $\mathrm{N}$ is prime or not, using position calculation formulas, as follows:

Divisibility by:

$(3000 \times \mathrm{n}+7) \times(3000 \times \mathrm{n}+901) \mathrm{F}=2$

$7 \times \mathrm{n} ; 901 \times \mathrm{n} ; 901+3 \quad 007 \mathrm{xn} ; 901 \mathrm{x} 2+6 \quad 007 \mathrm{xn} ; 901 \mathrm{x} 3+9$ 007xn;

$7 \mathrm{xn}$ correspond to: $7 \times(3000 \times \mathrm{n}+901) ; 901 \times n$ correspond to: 901 $\times(3000 \times \mathrm{n}+7)$;

$901+3007 x n$ correspond to: $3007 \times(3000 \times n+901)$;
$901 \times 2+6007 x n$ correspond to: $6007 \times(3000 \times n+901)$;

901x3+9 007xn correspond to: $9007 \times(3000 \times \mathrm{n}+901)$;

If not results indicate position of $\mathrm{N}$ decreased by the factor $\mathrm{F}=2$, the number studied does not divide with multiples of 3000 plus pair of numbers 7-901

$(3000 \times \mathrm{n}+307) \times(3000 \times \mathrm{n}+3001) \mathrm{F}=307$ $307 \mathrm{xn}$

$307 \times \mathrm{n} ; 3001 \times \mathrm{n} ; 3001+3$ 307xn; $3001 \times 2+6307 x n ; 3001 \times 3+9$

$307 \times \mathrm{n}$ correspond to: $307 \times(3000 \times \mathrm{n}+3001) ; 3001 \times \mathrm{n}$ correspond to: $3001 \times(3000 \times \mathrm{n}+307)$;

$3001+3307 \times \mathrm{n}$ correspond to: $3307 \times(3000 \times \mathrm{n}+3001)$;

$3001 \times 2+6307 x n$ correspond to: $6307 \times(3000 \times n+3001)$;

$3001 \times 3+9307 x n$ correspond to: $9307 \times(3000 \times n+3001) ; \ldots$

Extract factor $\mathrm{F}=307$ out of the position number of $\mathrm{N}$ than check calculation above.

$(3000 \times \mathrm{n}+607) \times(3000 \times \mathrm{n}+2101) \mathrm{F}=425$

$607 \times n ; 2101 \times n ; 2101+3607 x n ; 2101 \times 2+6607 x n ; 2101 \times 3+9$ 607xn;

Or,

$(3000 \times \mathrm{n}+2707) \times(3000 \times \mathrm{n}+1801) \mathrm{F}=1625$

$2707 \times \mathrm{n} ; 1801 \times \mathrm{n} ; 1801+5707 \mathrm{xn} ; 1801 \times 2+8707 \mathrm{xn} ; 1801 \times 3+11$ $707 \mathrm{xn}$;

If none of the operations related to 400 factors do not give as results the position of studied number, this number is prime.

For this example ( $p=359612$ ) we check these calculations:

Divisibility by:

$(3000 \times \mathrm{n}+7) \times(3000 \times \mathrm{n}+901) \mathrm{F}=2 \mathrm{P}-\mathrm{F}=359610$

$7 \times 51372=359604$ not divisible by $7 \times(3000 \times n+901)$

$901 \times 399=359499$ not divisible by $901 \times(3000 \times n+7)$

$901+3007 \times 119=358734-/ /-3007 \times(3000 \times \mathrm{n}+901)$

$901 \times 2+6007 \times 59=356215-/ /-6007 \times(3000 \times \mathrm{n}+901$

$901 \times 3+9007 \times 39=353976-/ /-9007 \times(3000 \times n+901)$

$901 \times 4+12007 \times 29=351807-/ /-12007 \times(3000 \times \mathrm{n}+901)$

$901 \times 5+15007 \times 23=349666-/ /-15007 \times(3000 \times n+901)$

$901 \times 6+18007 \times 20=365546-/ /-18007 \times(3000 \times \mathrm{n}+901)$

$901 \times 7+21007 \times 16=342419-/ /-21007 \times(3000 \times n+901)$

$901 \times 8+24007 x 14=343306-/ /-24007 \times(3000 \times \mathrm{n}+901)$

$901 \times 9+27007 \times 13=359200-/ /-27007 \times(3000 \times n+901)$

$901 \times 10+30007 \times 11=339087-/ /-30007 \times(3000 \times n+901)$

$901 \times 20+60007 \times 5=318055-/ /-60007 \times(3000 \times n+901)$

$901 \times 30+90007 \times 3=297054-/ /-90007 \times(3000 \times n+901)$

$901 \times 40+120007 \times 2=276054-/ /-120007 \times(3000 \times n+901)$

$901 \times 50+150007 \times 2=345064-/ /-150007 \times(3000 \times \mathrm{n}+901)$ 
$901 \times 60+180007 \times 1=234067-/ /-180007 \times(3000 \times n+901)$

$901 \times 92+276007=358899-/ /-276007 \times(3000 \times n+901)$

Last calculation can be performed.

Testing for number $\mathrm{N}$ continues with:

Divisibility by:

$(3000 \times n+37) \times(3000 \times n+1711) F=21 P-F=359591$

$(3000 \times \mathrm{n}+67) \times(3000 \times \mathrm{n}+721) \mathrm{F}=16 \mathrm{P}-\mathrm{F}=359596$

Divisibility by:

$(3000 \times \mathrm{n}+2999) \times(3000 \times \mathrm{n}+2693) \mathrm{F}=2692 \mathrm{P}-\mathrm{F}=356920$

$2999 \times 119=356881-/ /-2999 \times(3000 \times n+2693)$

$2693 \times 132=355476-/ /-2693 \times(3000 \times n+2999)$

$2693+5999 \times 59=356634-/ /-5999 \times(3000 \times \mathrm{n}+2693)$

$2693 \times 2+8999 \times 39=356347-/ /-8999 \times(3000 \times n+2693)$

$2693 \times 10+32999 \times 10=356920$, number identical to $\mathrm{P}-\mathrm{F}$,

So $\mathrm{N}$ is divisible by 32999 .

\section{For termination five digits}

The calculation algorithm is:

Pas.1: Determine the position number and column it belongs;

Pas.2: Last five digits of the calculated number indicates the termination position of tested number;

Pas 3: Determine factors for termination and column number tested. I have illustrated the calculation of factors termination 00110 , column 1;

Pas.4: We divisibility test the formulas for calculating factorial.

Positions calculated results do not contain termination 00110

For pair of numbers $31-397$

$31 \times(3000000 \mathrm{xn}+1161397) \mathrm{p}=12+31 \times \mathrm{n}$; divisibility by 31

$3031 \times(3000000 \mathrm{xn}+1800$ 397) $\mathrm{p}=1819+3031 \times \mathrm{n}-/ /-3031$ 031

$6031 \times(3000000 x n+2439397) \mathrm{p}=1819+3085+6031 \times \mathrm{n}-/ /-6$

$9031 \times(3000000 x n+3078397) p=1819+3085 \times 2+1278+9031$ $\times \mathrm{n}-/ /-9031$

$12031 \times(3000000 \times n+3717397) \mathrm{p}=1819+3085 \times 3+1278 \times$ (2)!+12 $031 \times \mathrm{n}-/ /-12031$

$15031 \times(3000000 x n+4356379) \mathrm{p}=1819+3085 \times 4+1278 \times$ (3)!+15 $031 \times \mathrm{n}-/ /-15031$

$18031 \times(3000000 \mathrm{xn}+4995379) \mathrm{p}=1819+3085 \times 5+1278 \times$ (4)!+18031× n -//- 18031

$2997031 \times(3000000 \times n+639522379) p=1819+3085 \times 998+1278$ $\times(997) !+2997031 \times \mathrm{n}$ divisibility by 2997031

$3000031 \times(3000000 x n+640161379) \mathrm{p}=1819+3085 \times 999+1278$ $\times(998) !+3000031 \times n$ divisibility by 3000031

$3003031 \times(3000000 \times n+640800379) \mathrm{p}=1819+3085 \times 1000+1$ $278 \times(999) !+3003031 \times \mathrm{n}$ divisibility by 3003031
And,

$397 \times(3000000 x n+2403031) p=318+397 \times n$ divisibility by 397

$3397 \times(3000000 \mathrm{xn}+234031) \mathrm{p}=265+3397 \times \mathrm{n}-/ /-3397$

$6397 \times(3000000 x n+1065031) \mathrm{p}=265+2006+6397 \times \mathrm{n}-/ /-6397$

$9397 \times(3000000 x n+1896031) \mathrm{p}=265+2006 \times 2+1662+9397 \times$ n $-/ /-9397$

$12397 \times(3000000 \times n+2727031) \mathrm{p}=265+2006 \times 3+1662 \times(2) !+12$ $397 \times \mathrm{n}-/ /-12397$

$15397 \times(3000000 x n+3558031) p=265+2006 \times 4+1662 \times(3) !+15$ $397 \times \mathrm{n}-/ /-15397$

$18397 \times(3000000 x n+4389031) \mathrm{p}=265+2006 \times 5+1662 \times(4) !+18$ $397 \times \mathrm{n}-/ /-18397$

$2997397 \times(3000000 x n+829572031) \mathrm{p}=265+2006 \times 998+1662$ $\times(997) !+2997397 \times \mathrm{n}$ divisibility by 2997397

$3000397 \times(3000000 x n+830403031) p=265+2006 \times 999+1662$ $\times(998) !+3000397 \times \mathrm{n}$ divisibility by 3000397

$3003397 \times(3000000 \times n+831234031) \mathrm{p}=265+2006 \times 1000+1662$ $\times(999) !+3003397 \times \mathrm{n}$ divisibility by 3003397

Or, pair of numbers $331-1297$

$331 \times(3000000 \times n+2755297) \mathrm{p}=304+331 \times \mathrm{n}$ divisibility by 331

$3331 \times(3000000 \mathrm{xn}+994297) \mathrm{p}=1104+3331 \times \mathrm{n}-/ /-3331$

$6331 \times(3000000 x n+2233297) p=1104+3609+6331 \times n-/ /-6$ 331

$9331 \times(3000000 x n+3472297) p=1104+3609 \times 2+2478+9331$ $\times \mathrm{n}-/ /-9331$

$12331 \times(3000000 \mathrm{xn}+4711297) \mathrm{p}=1104+3609 \times 3+2478 \times$

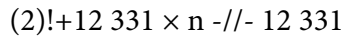

$15331 \times(3000000 \times n+5950297) p=1104+3609 \times 4+2478 \times$ (3)!+15 $331 \times \mathrm{n}-/ /-15331$

$18331 \times(3000000 \mathrm{xn}+7189297) \mathrm{p}=1104+3609 \times 5+2478 \times$

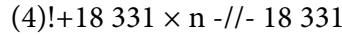

And,

$1297 \times(3000000 \times n+342331) \mathrm{p}=148+1297 \times \mathrm{n}$ divisibility by 1297

$4297 \times(3000000 x n+1773$ 331) p=2 540+4 $297 \times \mathrm{n}-/ /-4297$ 297

$7297 \times(3000000 x n+3204331) \mathrm{p}=2540+5254+7297 \times \mathrm{n}-/ /-7$

$10297 \times(3000000 x n+4635331) p=2540+5254 \times 2+2862+10$ $297 \times \mathrm{n}-/ /-10297$

$13297 \times(3000000 x n+6066331) \mathrm{p}=2540+5254 \times 3+2862 \times$ (2)!+13 $297 \times \mathrm{n}-/ /-13297$

$16297 \times(3000000 \mathrm{xn}+7497331) \mathrm{p}=2540+5254 \times 4+2862 \times$ (3)!+16 $297 \times \mathrm{n}-/ /-16297$

$19297 \times(3000000 \mathrm{xn}+8928331) \mathrm{p}=2540+5254 \times 5+2862 \times$ (4)!+19297 × n -//- 19297

\section{Conclusion}

Number testing is done with all the 400 pairs of numbers in the 
group factorial. Factorial multiplication process has as principle of calculation pairs of numbers that belong to the factorial group unique to each termination and column.

\section{References}

1. Canfield ER, Erdos P, Pomerance C (1983) On a problem of Oppenheim concerning Factorisatio Numerorum. J Number Theory 17: 1-28.

2. Davis JA, Holdridge DB (1983) Factorization sings the quadratic sieve algorithm. Advances in Cryptology 2: 103-113.
3. Lehmer DH, Powers RE (1931) on factoring large numbers. Bull her Math Soc 37: 770-776.

4. Miller JCP (1975) on factorisation with a suggested new approach. Math Comp 29: $155-772$.

5. Pomerance C, Wagstaff SS (1983) Implementation of the continued fraction algorithm. Cow Numerantium 37: 99-118.

6. Morrison MA, Brillhart J (1975) A method of factoring and the factorization of $F_{7}$. Math Comp 29: 183-205. 